\title{
Immune biomarker-based enrichment in sepsis trials
}

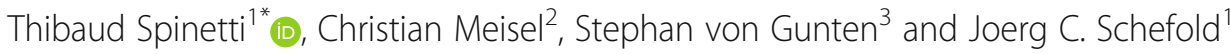

\section{Dear Editor}

We read with great interest the study by Anderson et al. recently published in Critical Care [1]. The study assessed whether soluble mediators (IL-8, sTNFR1, and Ang-2) could be used as biomarkers to "enrich" subject populations with higher mortality risk in subsequent clinical trials. The authors addressed "immunocompetence" of patients using clinical parameters (APACHE-II score and/or presence of ARDS). They found that both IL-8 and sTNFR1 (but not Ang-2) were suitable for identification of patients with higher mortality risk and concluded that IL-8 and sTNFR1 can be used as "prognostic enrichment factors" in future clinical sepsis studies.

Biomarker-based prognostic enrichment appears important to select sample populations with a greater likelihood of having improved clinical outcomes following a given therapeutic intervention. Although sTNFR1 and IL8 levels may be associated with higher mortality in certain sepsis patients, however, selection of the correct "enrichment markers" should be performed cautiously and based on a solid underlying biological rationale. This may, for example, be of particular importance in the field of clinical sepsis trials testing immunomodulatory interventions where assessment of the pleiotropic cytokine IL-8 would likely introduce considerable bias and should thus not be used to stratify respective patient populations. Failure of an adequate peri-interventional characterization may at least partly explain the failure of a number of previous sepsis trials testing immunological interventions (e.g., corticosteroids, strategies testing antiTNF or anti-LPS). In the study by Anderson et al., "immunocompetency" in sepsis patients was defined using clinical criteria. However, it seems that immunocompetency, i.e., (functional) immune phenotype, cannot be assessed by predominantly clinical parameters and

\footnotetext{
* Correspondence: thibaud.spinetti@insel.ch

This comment refers to the article available at https://doi.org/10.1186/ s13054-019-2684-2.

${ }^{1}$ Department of Intensive Care Medicine, Inselspital, Bern University Hospital, University of Bern, Bern, Switzerland

Full list of author information is available at the end of the article
}

should be based on comprehensive functional immune markers (e.g., mHLA-DR expression [2-4]) in order to identify individuals who would benefit most from a given intervention.

We are well aware that the focus of the article was to address the important question of whether biomarkerbased enrichment would lead to better stratification of future trial cohorts.

While we appreciate the insights provided by Anderson et al., we believe that it will be crucial (and challenging) to continue the quest for the "correct" enrichment markers to succeed in the design of novel therapeutic interventions, which may require more extensive reverse translational research and personalized treatment approaches [5]. In the light of failure of a large number of previous clinical sepsis trials, it seems apparent that biomarker enrichment using appropriate mediators is needed and may open several avenues towards more personalized treatment approaches in sepsis.

\section{Abbreviations \\ IL-8: Interleukin 8; sTNFR1: Soluble tumor necrosis factor receptor-1; Ang- 2: Angiopoietin-2; APACHE : Acute Physiology, Age, Chronic Health Evaluation; ARDS: Acute Respiratory Distress Syndrome; TNF: Tumor necrosis factor; LPS: Lipopolysaccharide}

\section{Acknowledgements}

Not applicable.

\section{Authors' contributions}

TS and JCS drafted and finalized the manuscript. CM and SVG helped to draft the manuscript and revised the manuscript for important intellectual content. All authors read and approved the final version of the manuscript.

\section{Authors' information}

Not applicable.

\section{Funding}

Not applicable.

\section{Availability of data and materials Not applicable.}

\section{Ethics approval and consent to participate Not applicable.}

\section{Consent for publication \\ Not applicable.}




\section{Competing interests}

TS and JCS reports grants (full departmental disclosure) from Orion Pharma, Abbott Nutrition International, B. Braun Medical AG, CSEM AG, Edwards Lifesciences Services GmbH, Kenta Biotech Ltd, Maquet Critical Care AB, Omnicare Clinical Research AG, Nestle, Pierre Fabre Pharma AG, Pfizer, Bard Medica S.A., Abbott AG, Anandic Medical Systems, Pan Gas AG Healthcare, Bracco, Hamilton Medical AG, Fresenius Kabi, Getinge Group Maquet AG, Dräger AG, Teleflex Medical GmbH, Glaxo Smith Kline, Merck Sharp and Dohme AG, Eli Lilly and Company, Baxter, Astellas, Astra Zeneca, CSL Behring, Novartis, Covidien, Philips Medical, Phagenesis Ltd, Prolong Pharmaceuticals, and Nycomed outside the submitted work. The money went into departmental funds. No personal financial gain applied. The other authors declare that they have no competing interests.

\section{Author details}

'Department of Intensive Care Medicine, Inselspital, Bern University Hospital, University of Bern, Bern, Switzerland. ${ }^{2}$ Department of Medical Immunology, Charité University Medicine Berlin, Berlin, Germany. ${ }^{3}$ Institute of

Pharmacology, University of Bern, Bern, Switzerland.

Received: 23 January 2020 Accepted: 11 February 2020

\section{Published online: 19 February 2020}

\section{References}

1. Anderson BJ, Calfee CS, Liu KD, Reilly JP, Kangelaris KN, Shashaty MGS, Lazaar AL, Bayliffe Al, Gallop RJ, Miano TA, et al. Plasma sTNFR1 and IL8 for prognostic enrichment in sepsis trials: a prospective cohort study. Crit Care. 2019;23(1):400.

2. Pfortmueller CA, Meisel C, Fux M, Schefold JC. Assessment of immune organ dysfunction in critical illness: utility of innate immune response markers. Intensive Care Med Exp. 2017;5(1):49.

3. Monneret G, Lepape A, Voirin N, Bohe J, Venet F, Debard AL, Thizy H, Bienvenu J, Gueyffier F, Vanhems P. Persisting low monocyte human leukocyte antigen-DR expression predicts mortality in septic shock. Intensive Care Med. 2006;32(8):1175-83.

4. Schefold JC. Measurement of monocytic HLA-DR (mHLA-DR) expression in patients with severe sepsis and septic shock: assessment of immune organ failure. Intensive Care Med. 2010;36(11):1810-2.

5. von Gunten $\mathrm{S}$. The future of pharmacology: towards more personalized pharmacotherapy and reverse translational research. Pharmacology. 2019; 105(1-2):1-2.

\section{Publisher's Note}

Springer Nature remains neutral with regard to jurisdictional claims in published maps and institutional affiliations. 